\title{
Filigrane
}

Écoutes psychothérapiques

\section{La résistance de la nature humaine}

\section{Martin Gauthier}

Volume 23, numéro 1, printemps 2014

Qu'est la psychanalyse devenue?

URI : https://id.erudit.org/iderudit/1026074ar

DOI : https://doi.org/10.7202/1026074ar

Aller au sommaire du numéro

Éditeur(s)

Revue Santé mentale au Québec

ISSN

1192-1412 (imprimé)

1911-4656 (numérique)

Découvrir la revue

Citer cet article

Gauthier, M. (2014). La résistance de la nature humaine. Filigrane, 23(1), 13-25. https://doi.org/10.7202/1026074ar

\section{Résumé de l'article}

Un écart s'est creusé entre la pratique pédopsychiatrique et la pratique psychanalytique depuis trente ans. Toute la psychothérapie a perdu en importance dans cette évolution qui ne concerne pas seulement les psychanalystes mais toute la société. Les changements culturels et sociaux, issus du système capitaliste marchand, apparaissent déterminants dans l'évolution des soins et le déclin de la psychanalyse. Cette dernière voudra résister aux côtés de la nature humaine qu'elle a participé à révéler et qui déborde le cadre réducteur qui lui est imposé par le postulat physicaliste.
Ce document est protégé par la loi sur le droit d'auteur. L'utilisation des services d'Érudit (y compris la reproduction) est assujettie à sa politique d'utilisation que vous pouvez consulter en ligne.

https://apropos.erudit.org/fr/usagers/politique-dutilisation/ 


\title{
La résistance de la nature humaine ${ }^{1}$
}

\author{
Martin Gauthier
}

Un écart s'est creusé entre la pratique pédopsychiatrique et la pratique psychanalytique depuis trente ans. Toute la psychothérapie a perdu en importance dans cette évolution qui ne concerne pas seulement les psychanalystes mais toute la société. Les changements culturels et sociaux, issus du système capitaliste marchand, apparaissent déterminants dans l'évolution des soins et le déclin de la psychanalyse. Cette dernière voudra résister aux côtés de la nature humaine qu'elle a participé à révéler et qui déborde le cadre réducteur qui lui est imposé par le postulat physicaliste.

«C'est une grosse économie de temps, dit le marchand [de pilules perfectionnées qui font disparaître la soif] [...] Les experts ont fait des calculs. On épargne cinquante-trois minutes par semaine». "Moi, se dit le petit prince, si j'avais 53 minutes à dépenser, je marcherais tout doucement vers une fontaine...» (SaintExupéry, 1946, 76).

\section{D'hier à aujourd'hui, de Budapest à Montréal}

etournons d'abord en 1918, à Budapest, alors que Freud parle avec enthousiasme "des voies nouvelles de la thérapeutique psychanalytique» (Freud, 1919). Il lance l'idée que «le nombre d'analystes s'accroisse à tel point que nous parvenions à traiter une foule de gens» (Freud, 1919, 140) dans des cliniques soutenues par l'état. Les traitements y seront gratuits et une quantité considérable du plomb de la suggestion directe s'y mêlera à l'or pur de l'analyse car «les diverses formes de maladies traitées par nous ne peuvent être guéries par une seule et même technique» (Freud, 1919, 139). Souvenons-nous du climat politique qui prévaut alors: l'Europe sort enfin de la pire guerre qu'elle avait connue jusque-là, la psychanalyse a attiré l'attention par son traitement des névroses de guerre qui cessent d'être conçues comme de la simple simulation, un généreux mécène hongrois soutient une première maison de publication psychanalytique, le vent de changement en Hongrie portera bientôt au pouvoir un nouveau gouvernement communiste 
qui fera une belle place à Ferenczi et à la psychanalyse. Ce gouvernement sera de courte durée, les forces contre-révolutionnaires le renverseront rapidement mais la psychanalyse deviendra l'un des trois grands courants de pensée qui marqueront le $\mathrm{XX}^{\mathrm{e}}$ siècle, avec le marxisme et le féminisme (Mahony, 1996). Ces trois courants s'inscriront comme des voies d'émancipation contre les forces oppressives, comme des chemins d'accès à une plus grande liberté.

De 1918, sautons presqu'un siècle plus tard, en novembre 2013. Je suis dans mon bureau à l'hôpital, devant mon écran d'ordinateur en visioconférence avec la directrice du programme Santé mentale-jeunesse d'un Centre de services sociaux et de santé (un CSSS, cette entité issue de la dernière réforme administrative québécoise). Nous partageons, elle et moi, une même responsabilité populationnelle, dans un régime étatique où les soins sont gratuits comme Freud l'imaginait. Cette directrice me parle d'un projet qui se met en place au CSSS : les usagers pourront éventuellement se brancher sur un portail internet par lequel ils ne feront plus seulement une demande écrite de services mais pourront être en visioconférence avec un intervenant, en temps réel, me dit-elle. Le pas suivant sera ensuite aisé: offrir des interventions thérapeutiques par ce créneau. «Que de temps et d'argent nous sauverons ainsi, tout en facilitant l'accès! », ajoute-t-elle, enthousiaste. Sur ce, notre communication se termine car j'ai une consultation familiale prévue. Je suis à imaginer ce que serait mon travail thérapeutique installé devant mon ordinateur en marchant vers la salle d'attente où les parents et leurs deux enfants sont tous captés par leurs tablettes et téléphones intelligents respectifs, isolément. Ils n'échangent pas un mot et semblent quitter à regret leur écran pour me suivre. Je marche devant eux en imaginant un choix de thérapie électronique à la carte pour tous les goûts.

\section{L'évolution de la psychothérapie: un fleuve sans sel}

À l'extrémité ouest de l'île d'Orléans, on dit l'eau douce tandis qu'à l'extrémité est, on la dit salée. Bien sûr, la quantité de sel se dissout progressivement dans les eaux des rivières qui rejoignent le fleuve et la réalité est plus nuancée et plus continue que celle des papilles gustatives. Imaginons néanmoins qu'il faille trente ans pour traverser l'île d'Orléans de bout en bout. Son extrémité salée est celle que j'ai connue dans le réseau public il y a trente ans, le sel psychanalytique n'étant alors pas le seul minéral mais certainement le plus abondant. Aujourd'hui, à l'extrémité douce, l'eau me semble de plus en plus sans sel aucun, et pas seulement sans sel analytique. Je vais chercher à vous expliquer cette impression d'un fleuve sans sel mais aussi faire valoir 
comment la nature humaine finit par s'imposer malgré les velléités de dessalement et d'homogénéisation.

Pendant les trente dernières années, ma vie professionnelle s'est divisée entre mon travail de psychanalyste en bureau privé et celui de pédopsychiatre dans un hôpital pédiatrique, l'Hôpital de Montréal pour enfants (HME). Un volet administratif s'est aussi ajouté dans la dernière décennie, d'abord au niveau analytique à la Société psychanalytique de Montréal et à la Société canadienne de psychanalyse, puis en tant que directeur du Département de psychiatrie du HME. Le département de psychiatrie du HME fut le premier département pédopsychiatrique universitaire fondé au Canada (Weiss, 1995). C'était en 1950, dans le grand chantier social de l'après-guerre. Quand on songe que les premières cliniques de guidance infantile ont été créées aux États-Unis seulement au début du Xx $\mathrm{Xx}^{\mathrm{e}}$ siècle en réponse aux besoins des jeunes délinquants amenés devant la cour, la pédopsychiatrie est une jeune discipline en Occident. Elle est apparue dans la foulée de forces sociales et politiques donnant une voix de plus en plus forte à l'individu, et, ce faisant, créant en quelque sorte l'enfance et l'adolescence. C'est là un autre fleuve, parti des Lumières, traversant la Révolution française, l'émergence de la thérapie morale et la rédaction des premières histoires de cas par Pinel, avant celle des grands romans du XIX siècle et l'avènement de la psychanalyse; il rejoint le Québec au sortir de la deuxième grande guerre. Depuis sa naissance institutionnelle en 1950, la pédopsychiatrie québécoise s'est développée en suivant l'évolution rapide de la société civile. Les forces sociales continuent d'influencer le devenir de la psychiatrie et je voudrais m'attarder à cette dimension socio-politique.

Quand j'arrive à l'Université McGill pour faire ma formation psychiatrique en 1980, la pensée psychanalytique y est l'inspiration centrale de la réflexion clinique et du travail thérapeutique, particulièrement au HME où les idées de Winnicott circulent déjà. En vérité, on ne fait ni n'enseigne la psychanalyse à proprement parler mais le travail puise largement aux hypothèses psychanalytiques. Les psychanalystes sont nombreux dans l'institution, de toute génération; les débats sont animés. Thérapie familiale beaucoup, thérapie de groupe et thérapie brève trouvent leur place à côté de la psychothérapie psychanalytique individuelle. La pharmacologie, encore peu utilisée en pédopsychiatrie - ce sera le cas jusqu'aux années 2000 - les approches comportementales, en émergence, et la recherche (notamment sur le trouble déficitaire de l'attention depuis les années 1960 au HME) complètent le tableau. Des principes développementaux guident l'organisation des soins 
pédopsychiatriques, ce qui implique des divisions administratives en fonction de l'âge et non des symptômes présentés.

Ces années-là n'étaient pas seulement salées dans l'institution psychiatrique. Dans la société civile, nombreux sont ceux qui promeuvent alors une démarche d'introspection comme source de mieux-être et de plus grande liberté. Des thérapies de tout ordre apparaissent, les sciences humaines ont le vent dans les voiles, les départements de médecine familiale en création incarnent un idéal de médecine globale et humaniste.

Encouragé par le milieu psychiatrique et l'organisation des soins d'alors, j'ai pu rapidement joindre les rangs de la Société de psychanalyse et construire ensuite ma pratique professionnelle sur deux pieds, l'un psychiatrique à l'hôpital et l'autre psychanalytique au bureau. Mon premier poste psychiatrique est à l'unité interne où nous admettons des enfants de moins de 13 ans, en moyenne pour plusieurs mois pendant lesquels j'y fais de la psychothérapie de jeu.

Si j'ai longtemps avancé sur deux pieds capables de faire des pas de danse ensemble, aujourd'hui, 30 ans plus tard, je fais le grand écart tant la distance s'est creusée entre psychiatrie et psychanalyse. Et à l'intérieur de la pédopsychiatrie s'est ajouté un écart entre santé mentale $1^{\text {re }}$ ligne et une psychiatrie hospitalière $2^{\mathrm{e}}$ ligne qui aspire, dans le milieu universitaire que je côtoie, à devenir tertiaire et quaternaire, voulant s'éloigner davantage de la première ligne et faire valoir un statut d'expert. Un exemple des transformations: l'unité interne où je travaillais n'est plus limitée aux préadolescents et la durée moyenne de séjour est maintenant de 10 jours. De thérapeutique, elle est devenue une unité dite de stabilisation et d'orientation.

Quand je songe à l'extrémité salée de mon parcours et compare le travail hospitalier actuel avec celui qui prévalait, une question surgit: "Où les familles et nous prenions-nous le temps? Où est-il passé depuis, tout ce temps que nous avions?» Car l'expérience du temps est si différente d'une époque à l'autre! Tout s'est tellement accéléré alors que les problèmes cliniques ont gagné en nombre et en complexité tandis que les ressources professionnelles ont diminué. Le travail hospitalier n'est plus le même. Ce n'est pas tant que d'autres modèles psychothérapeutiques ont supplanté le modèle analytique, c'est une transformation majeure du cadre de travail qui a eu lieu. La psychothérapie est devenue au mieux secondaire, au pire négligeable alors que la pharmacologie domine et l'intervention est pressée. La discontinuité et l'inaccessibilité des services entraînent une détérioration de l'état des patients, les soins s'orientent de plus en plus vers l'intervention de crise au sein d'une 
prise en charge concrète et ponctuelle. Dans l'urgence, la pensée rétrécit, le geste prévaut, la fragmentation isole.

Trente ans plus tard, le patient n'est plus le même, les professionnels et la société non plus. Celle-ci valorise maintenant l'expert-consultant qui pose un diagnostic, dicte une conduite à suivre et ne s'encombre pas de la continuité des soins. Ce qui est considéré lourd et coûteux, mon expérience administrative me l'a bien appris à l'heure de la méthode Lean ${ }^{2}$, est d'ailleurs toute relation qui dure. Dans cette évolution, on a fait taire les psychiatres en les payant grassement pour l'évaluation d'un maximum de cas le plus rapidement possible. Tout le système semble organisé comme une vaste défense contre les rapports affectifs qui impliqueraient temps et continuité, avec l'équation que la relation entraîne dépendance et coûts excessifs.

La position du professionnel n'est plus la même face à la souffrance: nous restons maintenant davantage en périphérie, nous sommes observateurs et nous agissons de l'extérieur. L'intériorité est vue avec méfiance et tenue à distance, l'intervention prend la voie chimique et éducative. Les mots ont perdu en pouvoir autre que suggestif, la relation a cédé le pas aux actes. Et cela se passe également du côté des patients : ceux-ci veulent rarement s'ouvrir ; leur attente en est une d'un geste qui guérit, d'un expédient, d'une potion. Eux aussi sont pressés, le temps manque. Nombreux sont les parents ou les professeurs qui réclament une médication qu'ils obtiendront d'un autre si vous leur refusez. Organiser un rendez-vous familial devient souvent un tour de force dans l'agenda de chacun.

J'exagère le trait car il y a encore un peu de psychothérapie à l'hôpital, cognitico-comportementale, psycho-dynamique ou éclectique, avec essentiellement l'objectif de soutenir le moi et permettre rapidement la réduction des symptômes les plus importants. Reste que la psychothérapie a globalement perdu beaucoup de terrain et c'est cette position si précaire que je compare à l'absence de tout sel dans l'eau. Je force le trait pour mettre en relief un courant de fond qui me semble déterminant dans l'évolution observée. Ce courant n'est pas d'abord un autre modèle psychothérapeutique mais une idéologie sociale plus vaste et plus insidieuse.

Le HME a un service d'urgence achalandé qui colore tout son fonctionnement actuel. Sans doute n'est-il pas l'hôpital le plus représentatif du réseau québécois. La première ligne de soins, les CSSS, demeure certes un lieu où il se fait davantage de psychothérapie en milieu public, quoique la réalité varie d'un établissement à l'autre. Mais il reste que globalement toute la psychothérapie bat de l'aile. 


\section{La psychanalyse au sein des forces sociales}

Devant le recul de la pensée analytique, on a pu reprocher aux psychanalystes leur retrait des institutions et leur silence sur la place publique. On constate aussi qu'il y a eu une fracture générationnelle, que les jeunes professionnels ne se sont plus tournés vers la psychanalyse comme leurs aînés l'avaient fait. Les sociétés de psychanalyse vieillissent, la littérature psychanalytique décroît dramatiquement car elle ne vend plus, les signes de déclin se multiplient. Faut-il blâmer les psychanalystes pour leur devenir et pour celui de nos institutions psychiatriques? Cela me semble fort insuffisant comme explication. Car la psychanalyse appartient au champ social et est sujette aux mêmes forces complexes qui façonnent toute notre société. Ce n'est pas seulement la psychanalyse qui n'est plus ce qu'elle était, la culture n'est plus la même. Je questionne un courant de fond participant, de manière prépondérante, aux changements survenus dans nos institutions de soins psychiatriques, à la dévalorisation des sciences humaines en général, à la transformation de la psychopathologie et au déclin de la psychanalyse. Je vous invite à considérer les choses politiquement, avec le déclin de la psychanalyse comme symptôme d'un plus grand syndrome social.

Mon constat se situe dans la foulée de la thèse de l'historien Eli Zaretsky (2004), professeur à la New School for Social Research de New York. Celuici compare le rôle de la psychanalyse pendant la deuxième révolution industrielle à celui du calvinisme pendant le développement du capitalisme lors de la première révolution industrielle. L'une et l'autre, psychanalyse et calvinisme, participèrent ainsi à la montée des forces capitalistes qui finirent par les reléguer à l'arrière-plan et même par s'opposer à eux. Calvinisme et psychanalyse prirent leur essor dans un terreau social particulier où ils ouvrirent une voie d'abord subversive. La psychanalyse a ainsi marché un temps côte à côte avec les forces capitalistes qui profitaient des revendications individuelles que la nouvelle science soutenait tandis que cette dernière profitait de la nouvelle richesse économique qui donnait temps et soif de liberté. Mais la psychanalyse ne sert plus, le capitalisme a évolué de telle manière à s'opposer à ses visées introspectives. Le matérialisme ambiant, la révolution virtuelle et le commerce mondialisé font miroiter la liberté non plus dans un regard sur soi mais dans la nouvelle extériorité où se bâtit maintenant le monde. L'espace virtuel, sous un vent de marchandisation, transforme de plus en plus l'espace mental et l'espace relationnel, balisant un futur encore difficile à cerner au présent ${ }^{3}$, où la psychanalyse trouve difficilement place. 
La lecture que propose Zaretsky à la lumière de l'évolution de nos sociétés occidentales s'arrête aux années 1960. Il voit alors déjà les signes de déclin de la psychanalyse avec la montée de la société postindustrielle assise sur les services et la technologie. En soutien de sa thèse, il est intéressant de constater qu'actuellement, les lieux dans le monde où la psychanalyse prend de l'essor sont ceux qui commencent à jouir d'une plus grande aisance économique et d'une nouvelle liberté de penser, comme en Chine, en Amérique du Sud et dans les pays de l'ancien bloc soviétique. À l'opposé, sans doute faut-il se demander comment évolue notre liberté de pensée dans nos pays plus riches et porteurs d'une plus longue histoire démocratique.

Je mentionnais plus tôt l'importance de trois grands courants de pensée au $\mathrm{XX}^{\mathrm{e}}$ siècle, marxisme, féminisme et psychanalyse, mais un quatrième a poursuivi son chemin comme une grosse bête qui se nourrit de tous les autres. En fait moins celui d'une bête, le capitalisme a pris plus que jamais le visage impersonnel de la machine. Son grand allié, que Max Weber (1920) avait tôt reconnu, est la bureaucratie. La bureaucratisation de la vie sociale a aujourd'hui infiltré tout le tissu de nos vies (Kundera, 2005). Elle utilise la nouvelle technologie pour soutenir ses visées. Notre rapport au monde et au temps s'en trouvent transformés.

Je ne veux pas diaboliser notre société marchande mais il me semble important d'en reconnaitre les conséquences sur la pensée, sur nos choix de société, sur l'offre et la demande de soins. Ces conséquences ne sont pas faciles à cerner dans l'immédiat car nous avons le nez dessus, nous y baignons. Toute idéologie cherche à contrôler la pensée au service de sa vision du monde. Les quelques traits que je dessine mériteraient un plus long développement et des nuances.

Aujourd'hui, avec la complexification des connaissances, «[o]n ne veut plus le savoir, on veut le voir», prophétisait Yvon Deschamps. On veut des évidences ${ }^{4}$, nous nous voulons evidence-based. En adoptant le présupposé physicaliste $^{5}$ comme modèle unique de scientificité dans un but de rationalité instrumentale ${ }^{6}$, on a trouvé un outil simplificateur et réducteur au service du contrôle. C'est une nature humaine particulière que trace ce postulat physicaliste, une nature humaine conçue comme organisée par des lois naturelles immuables. Le comportement humain y apparaît invariablement soumis à des causes identifiables que l'on peut quantifier. On aborde alors la santé mentale à partir d'une position d'observateur extérieur et la prise d'un médicament y est devenue le paradigme central du jugement d'efficacité. Les autres approches thérapeutiques sont évaluées dans cette perspective et se 
veulent de plus en plus standardisées dans un souci d'homogénéité et de reproductivité. Dans cette foulée, le facteur humain (Dejours, 2007) pose problème ${ }^{7}$. Il fait figure d'imprévisible qui contamine ce qui se veut pur et maîtrisé.

La méthode expérimentale prête son objectif de reproductibilité contrôlée à l'appareil bureaucratique et aux visées marchandes. L'homogénéisation favorise la maîtrise, tandis que la marchandisation et son rétrécissement de l'espace subjectif transforment le rapport au temps. Le capitalisme actuel impose un régime addictif qui fonctionne par la création de nouveaux besoins se substituant toujours davantage aux relations affectives. Le rapport à la gratification a été modifié alors que tombent les structures plus secondarisées, notamment avec la transformation des structures familiales et une dévalorisation des processus sublimatoires. L'efficacité actuelle passe par le plus immédiat possible tout en niant la carence affective que cela engendre. La technologie virtuelle, elle aussi d'abord révolutionnaire et promettant une liberté accrue, est en voie de voir ses buts être pervertis au service des processus primaires et d'un raccourci du travail de pensée, sans négliger tous les problèmes de confidentialité et de contrôle social insidieux. Cette technologie avance rapidement, sans que nous ayons le temps d'en saisir tous les enjeux. Les soins virtuels que j'évoquais plus tôt ont d'ailleurs déjà débuté.

\section{Un avenir de la psychanalyse dans la résistance de la nature humaine}

Notre rapport au monde passe nécessairement par une médiation ou une instrumentation, ce qui peut être un outil comme une machine ou un langage. Ainsi, ce rapport est toujours technique et toute technique est culturelle; elle s'inscrit nécessairement dans la culture qu'elle contribue à transformer. Toute technique implique aussi un jugement d'efficacité à transformer le monde réel, jugement qui évolue à mesure que le monde et la culture se transforment (ce que Zaretsky abordait en soulignant la complicité temporaire de la psychanalyse et de la deuxième révolution industrielle).

La psychanalyse est elle-même une technique. Elle fut une révélation par le nouveau rapport qu'elle ouvrait sur le monde, sur les autres et sur soimême. Toute nouvelle technique est une nouvelle fenêtre sur le monde. Kundera (2005) dit cela de l'invention du roman avec Rabelais, Cervantès et Fielding: «[Cette invention] découvre un aspect jusqu'alors inconnu, caché, de la nature humaine» (Kundera, 2005, 19). La psychanalyse a été un grand outil de découverte et la nature humaine n'est plus la même à nos yeux depuis 
son invention. Notre culture en porte la trace mais comment l'assimile-t-elle, comment la digère-t-elle progressivement?

Les soins psychiatriques actuels veulent régimenter la nature humaine que la psychanalyse a participé à révéler. Cette nature humaine bouleverse l'autre nature, celle des lois physiques prédéterminées et immuables (Laplanche, 2007). Winnicott y a consacré son œuvre. En introduisant son livre intitulé La nature humaine (1988), il définissait ainsi son projet (je le cite en anglais car la traduction française disponible ${ }^{8}$ ne rend pas justice ni au sens ni à la musique lexicale du texte original) : «I shall hope to make clear how first from a primary merging of the individual with the environment comes an emergence, the individual staking a claim, becoming able to be in a world that is disclaimed (je souligne)» (Winnicott, 1988,8). L'individu en émergence, en invention, revendique; il établit son droit et sa capacité à être dans un monde dont il se sépare, qui ne lui appartient plus en propre.

$\mathrm{Si}$, avec Winnicott, la nature humaine vit dans l'émergence et la revendication de l'être, où en sommes-nous aujourd'hui? Quel être revendiquonsnous? De quelle nature humaine nous réclamons-nous et inventons-nous? La psychanalyse peut-elle encore faire rêver de liberté au sein de notre société?

La psychanalyse n'est plus dans les goûts du temps même si elle a été un grand outil d'émancipation personnelle et sociale. Si elle est devenue aujourd'hui plus marginale, tenue comme moins efficace et empêtrée dans ses anciennes manières, la réalité qu'elle veut dégager ne disparaîtra pas pour autant. La nature humaine dans sa complexité résiste. Et c'est dans cette résistance que je trouve espoir en tant que psychanalyste dans un milieu hospitalier si peu accueillant: la nature humaine déborde, crie sa souffrance, ne rentre pas dans les cadres établis dans lesquels on veut la réduire. La psychiatrie, ses professionnels et ses patients ne vont pas bien sans l'espace d'une parole qui revendique, sans temps relationnel, sans fenêtre pour l'être.

On sait combien, dans toute analyse et toute thérapie, les moules préexistants ne servent plus, qu'il faut chaque fois réinventer, adapter l'outil à la tâche. C'est le bonheur et la misère de la chose: toute véritable thérapie est transgressive, avançant à partir des échecs de la technique et du savoir. Depuis Kant, on a donné le nom de réel, en contraste à la réalité, à cette part du monde qui se fait connaître par sa résistance à la maîtrise technique et à la connaissance scientifique. C'est la partie de la réalité qui résiste à la symbolisation, celle toujours en excès, qui force à l'inventivité et à l'ingéniosité. On peut parler de notre activité subjectivante pour nommer ce travail de 
symbolisation qu'exige notre rapport au réel, travail toujours dépassé, à poursuivre, ouvert. La vie se trouve là et nous entraîne là, aux marges de la réalité, dans ce qui résiste.

La psychanalyse est née de l'exploration par Freud de ce qui faisait résistance à la technique qu'il employait à ses débuts. C'est ensuite aux marges de la résistance que la psychanalyse a avancé. Et c'est encore dans le travail de la résistance que la pensée psychanalytique a un avenir. Elle cesse d'avoir un horizon quand elle pense savoir et n'explore plus. Le système de soins actuel se bute de plus en plus à ses limites; il ne peut bannir la nature humaine sans que celle-ci ne se fasse entendre. La psychanalyse trouvera toujours son meilleur allié dans cette nature dont elle cherche à traduire la complexité et la nécessaire subjectivité.

Les patients déjouent toujours nos tentatives de les classifier et de les enfermer dans quelque chaîne; ils nous forcent à penser et c'est là que l'outil psychanalytique est de grand secours. La résistance est le grand écueil de toute technique thérapeutique. Il est d'ailleurs étonnant de voir les collègues utilisant d'autres paradigmes thérapeutiques chercher à ignorer son inévitable survenue, insister sur la motivation consciente des patients et souvent leur donner congé quand ils ne font pas ce qu'on attend d'eux, quand ils résistent.

Résistance de la nature humaine, résistance au carré de ceux qui se revendiquent d'elle, dans tous les milieux. Je qualifie ainsi de résistants les analystes travaillant encore dans nos institutions publiques mais ce ne sont pas les seuls. Sont également résistants tous ceux qui revendiquent encore la place de l'être dans les divers milieux cliniques et universitaires. Des foyers de résistance existent là où la pensée analytique inspire encore le travail qui s'y fait, à commencer par les bureaux privés qui peuvent sembler extérieurement plus douillets, ce qui n'est certes pas le cas au quotidien du transfert. La psychanalyse est partout un travail de la résistance, un travail de résistant face aux résistances individuelles et collectives.

La résistance se pose avant tout contre la pensée unique, contre un totalitarisme thérapeutique, contre la machine homogénéisante. La complexité des enjeux exige une diversité des moyens et des approches, avec place pour la relation et le travail de subjectivation.

Il y a un brin de romantisme à parler du psychanalyste résistant, ça flatte le narcissisme dans l'épreuve. Car le doute n'est jamais loin et les vents autodépréciateurs aisément levés dans l'environnement hospitalier peu hospitalier. D'où l'importance des lieux d'échange, des colloques, des revues, des 
associations et sociétés, comme des petits séminaires entre collègues : la psychanalyse résiste par la parole, l'être revendique sa place par un langage qui s'invente avec un autre. Les institutions psychanalytiques ne survivront certes pas sous leur forme actuelle mais il faudra toujours des lieux d'échanges entre ceux qui revendiquent un regard psychanalytique.

Cette voie implique un travail de pensée ouvert, sans nostalgie, pour ceux qui se réclament de l'héritage de Freud. De belles questions se posent face à notre époque, comme par exemple celle du cadre thérapeutique à l'heure du fast food, du speed dating et des pillules qui coupent les soifs. La psychanalyse tient toujours, et plus encore aujourd'hui, une position subversive face au temps mais il lui faut aussi penser sa technique au sein d'un environnement social où les repères changent beaucoup. Comment notre société évoluera-telle? Quel impact, par exemple, aura la place et le pouvoir social croissant des femmes, notamment en santé mentale? Que deviendrons-nous? La psychanalyse voudra mettre son grain de sel face aux changements sociaux qui nous emportent vers des rives marchandes et étroites.

\section{Conclusion: une question écologique}

En vérité, je cache assez bien le grand écart qui m’habite quand je marche devant une famille vers mon bureau. Celle dont je parlais plus tôt était composée du père, de la mère et leurs deux fils, et ils ne se doutaient de rien. Ils venaient voir le spécialiste qui allait mater un problème persistant, préciser le diagnostic du garçon de 17 ans et sûrement ajuster la médication. Depuis trois ans, ce grand adolescent se sent envahi de pensées obsessives qui l'empêchent de mener ses activités habituelles. Les pensées envahissantes sont elles-mêmes des scènes de pénétration et d'envahissement, particulièrement celle de chauves-souris porteuses de rage qu'il fait lui-même entrer par mégarde dans l'entre-toit de sa maison. Récemment une nouvelle crainte phobique s'est ajoutée : il pourrait, encore par mégarde, envoyer un message électronique menaçant à quelqu'un, même la nuit en dormant. Deux thérapies cognitivo-comportementales plus tard, l'ajout d'une médication, le déménagement des parents dans un voisinage sans chauve-souris, tout a visé à barricader la maison, à fermer les issues, à chasser la pensée indésirable qui résiste et s'infiltre malgré tout. Au contraire, vous vous doutez bien que le psychanalyste a voulu faire entrer les chauves-souris et les messages menaçants dans la pièce du bureau. Je me trouvais là à vouloir ouvrir les portes que les autres s'activaient à fermer. Et j'imaginais ainsi la survie de la psychanalyse, à l'heure marchande, comme une question écologique, un 
problème de sauvegarde de la nature humaine, celle que l'on veut chasser et qui heureusement résiste.

\author{
Martin Gauthier \\ 4000, avenue Marcil \\ Montréal (Québec) \\ H4A 2Z6 \\ martin.gauthier3@sympatico.ca
}

\title{
Notes
}

1. L'auteur présente ici le texte remanié de la conférence qu'il a donnée dans le cadre du Colloque organisé par la revue Filigrane, le 16 novembre 2013: «Qu’est la psychanalyse devenue?».

2. La méthode Lean (maigre, sans gras) repose sur une théorie de gestion de la production qui se veut sans gaspillage. Elle a pris origine chez Toyota au Japon et influence maintenant la gestion hospitalière du HME.

3. «La virtualisation ne relève donc pas de la déréalisation mais de la démultiplication des possibles ", écrit Dalsuet $(2013,34)$ qui constate par ailleurs l'instrumentalisation sociale des relations personnelles qu'internet favorise.

4. Évidence prend racine dans le voir. Le Robert définit l'évidence comme le «caractère de ce qui s'impose à l'esprit avec une telle force qu'il n'est besoin d'aucune preuve pour en connaître la vérité, la réalité». Un voir qui rend la pensée inutile...

5. Ce présupposé conçoit toute entité existante comme de nature physique et obéissant à des lois physiques. Il s'oppose au dualisme dont se réclame la psychanalyse.

6. Weber (1920) parle de «tournure utilitariste», qu'il associe aux principes moraux énoncés par Benjamin Franklin.

7. Je m'inspire dans ce qui suit de la pensée de Christophe Dejours dans son livre Le facteur humain, qui offre une belle synthèse des enjeux qui confrontent les sciences de l'homme au travail.

8. La traduction française aux Éditions Gallimard (Winnicott, 1990, 18) dit ceci : «J'espère pouvoir rendre clairement la façon dont, à partir d'une fusion primaire entre l'individu et l'environnement, quelque chose fuse, l'individu mettant en jeu ce qu'il veut et devenant capable d'exister dans un monde qui ne veut rien.»

\section{Références}

Dalsuet, A., 2013, T'es sur facebook? Qu'est-ce que les réseaux sociaux changent à l'amitié?, Paris, Flammarion Antidote.

Dejours, C., 2007, Le facteur humain, Paris, PUF Que sais-je?, 4e édition.

Freud, S., 1919, Les voies nouvelles de la thérapeutique psychanalytique, in La technique psychanalytique, PUF, Paris, 1953, 131-141.

Kundera, M., 2005, Le rideau, Paris, Gallimard Folio.

Laplanche, J., 2007, Nouveaux fondements pour la psychanalyse, Paris, PUF.

Mahony, P.J., 1996, Freud's Dora. A Psychoanalytic, Historical, and Textual Study, New Haven, Yale University Press.

Saint-Exupéry, A., 1946, Le Petit Prince, Paris, Gallimard. 
Weber, M.,1920, L'éthique protestante et l'esprit du capitalisme, Paris, Le Monde Flammarion, 2009.

Weiss, G., 1995, Child Psychiatry at McGill University and the Montreal Children's Hospital, in Building a Proud Past. 50 Years of Psychiatry at McGill, T.L. Sourkes \& G. Pinard eds, Montréal, McGill University.

Winnicott, D.W., 1988, Human Nature, London, Free Association Books.

Winnicott, D.W., 1990, La nature humaine, Paris, Gallimard.

Zaretsky, E., 2004, Le siècle de Freud. Une histoire sociale et culturelle de la psychanalyse, Paris, Albin Michel, 2008. 